\title{
THE PRIME FILTER THEOREM OF LATTICE IMPLICATION ALGEBRAS
}

\author{
YOUNG BAE JUN
}

(Received 23 March 2000)

\begin{abstract}
Using a special set $x^{-1} F$, we give an equivalent condition for a filter to be prime, and applying this result, we provide the prime filter theorem in lattice implication algebras.
\end{abstract}

2000 Mathematics Subject Classification. Primary 03G10, 06B10, 54 E15.

1. Introduction. In order to research the logical system whose propositional value is given in a lattice, $\mathrm{Xu}$ [3] proposed the concept of lattice implication algebras, and discussed some of their properties. Xu and Qin [4] introduced the notion of filters and implicative filters in a lattice implication algebra and investigated their properties. The present author [1] gave an equivalent condition of a filter and provided some equivalent conditions for a filter to be an implicative filter. Also, by using these results, an extension property for implicative filter was constructed. In [2], Liu and Xu defined the notion of prime filters and studied a decomposition theorem of lattice implication algebras.

In this paper, we first give an equivalent condition for a filter to be prime by using a special set $x^{-1} F$ and applying this result we provide the prime filter theorem in lattice implication algebras.

2. Preliminaries. First of all, we recall a few notions and properties.

By a lattice implication algebra we mean a bounded lattice $(L, \vee, \wedge, 0,1)$ with orderreversing involution "'” and a binary operation " $\rightarrow$ " satisfying the following axioms:

(I1) $x \rightarrow(y \rightarrow z)=y \rightarrow(x \rightarrow z)$,

(I2) $x \rightarrow x=1$,

(I3) $x \rightarrow y=y^{\prime} \rightarrow x^{\prime}$,

(I4) $x \rightarrow y=y \rightarrow x=1 \Rightarrow x=y$,

(I5) $(x \rightarrow y) \rightarrow y=(y \rightarrow x) \rightarrow x$,

(L1) $(x \vee y) \rightarrow z=(x \rightarrow z) \wedge(y \rightarrow z)$,

(L2) $(x \wedge y) \rightarrow z=(x \rightarrow z) \vee(y \rightarrow z)$,

for all $x, y, z \in L$.

In what follows the binary operation " $\rightarrow$ " will be denoted by juxtaposition. We can define a partial ordering " $\leq$ " on a lattice implication algebra $L$ by $x \leq y$ if and only if $x y=1$.

In a lattice implication algebra $L$, the following hold (see [3]):

(1) $0 x=1,1 x=x$, and $x 1=1$. 
(2) $x^{\prime}=x 0$.

(3) $x y \leq(y z)(x z)$.

(4) $x \vee y=(x y) y$.

(5) $x \leq y$ implies $y z \leq x z$ and $z x \leq z y$.

(6) $x \leq(x y) y$.

A subset $F$ of a lattice implication algebra $L$ is called a filter of $L$ if it satisfies:

(F1) $1 \in F$,

(F2) $x \in F$ and $x y \in F$ imply $y \in F$, for all $x, y \in L$.

Any filter $F$ of a lattice implication algebra $L$ has the property: if $x \leq y$ and $x \in F$, then $y \in F$.

3. The prime filter theorem. In the rest of this paper, the letter $L$ will be reserved, so far as is possible, for a lattice implication algebra.

Note that for a subset $F$ of $L$,

$$
\langle F\rangle=\left\{x \in L \mid a_{1}\left(a_{2} \cdots\left(a_{n} x\right) \cdots\right)=1 ; a_{1}, a_{2}, \ldots, a_{n} \in F\right\}
$$

is the smallest filter containing $F$ and is called the filter generated by $F$ (see [4]).

For any nonnegative integer $n$, we define $n(x) y$ recursively as follows: $0(x) y=y$, $1(x) y=x y$, and $(n+1)(x) y=x(n(x) y)$ for all $x, y \in L$. Using (I1) and (1) we know that $y(n(x) y)=1$, that is, $y \leq n(x) y$ for all $x, y \in L$.

Proposition 3.1. Let $F$ be a filter of $L$ and let $x \in L$. Then

$$
\langle F \cup\{x\}\rangle=\{y \in L \mid n(x) y \in F \text { for some nonnegative integer } n\}
$$

Proof. Let $y \in\langle F \cup\{x\}\rangle$. Then

$$
m(x)\left(a_{1}\left(a_{2} \cdots\left(a_{n} y\right) \cdots\right)\right)=1
$$

for some $a_{1}, a_{2}, \ldots, a_{n} \in F$ and some nonnegative integer $m$. Using (I1) repeatedly, we know that

$$
a_{1}\left(a_{2} \cdots\left(a_{n}(m(x) y)\right) \cdots\right)=1 .
$$

It follows from (F2) that $m(x) y \in F$ so that

$$
\langle F \cup\{x\}\rangle \subseteq\{y \in L \mid n(x) y \in F \text { for some nonnegative integer } n\} .
$$

Conversely, assume that $n(x) y \in F$ for some nonnegative integer $n$. It follows from $F \subseteq\langle F \cup\{x\}\rangle$ that $x((n-1)(x) y)=n(x) y \in\langle F \cup\{x\}\rangle$. Since $x \in\langle F \cup\{x\}\rangle$, we have $(n-1)(x) y \in\langle F \cup\{x\}\rangle$ by (F2). Repeating this process we know that $y=0(x) y \in$ $\langle F \cup\{x\}\rangle$. Hence

$$
\{y \in L \mid n(x) y \in F \text { for some nonnegative integer } n\} \subseteq\langle F \cup\{x\}\rangle,
$$

This completes the proof. 
DEFINITION 3.2. For any nonempty subset $F$ of $L$ and $x \in L$, we define

$$
x^{-1} F:=\{y \in L \mid x \vee y \in F\} .
$$

Note that if $F$ is a filter of $L$, then $1 \in x^{-1} F$.

Proposition 3.3. If $F$ is a filter of $L$, then $x^{-1} F$ is a filter of $L$ containing $F$.

Proof. Let $y \in x^{-1} F$ and $y z \in x^{-1} F$. Then $x \vee y \in F$ and $x \vee(y z) \in F$. Now

$$
(x \vee y)(x \vee z)=((y x) x)((z x) x) \geq(z x)(y x) \geq y z
$$

and $(x \vee y)(x \vee z) \geq x \vee z \geq x$. It follows that $x \vee(y z) \leq(x \vee y)(x \vee z)$ so that $(x \vee y)(x \vee z) \in F$. Using the fact that $F$ is a filter and $x \vee y \in F$, we get $x \vee z \in F$, that is, $z \in x^{-1} F$. This shows that $x^{-1} F$ is a filter of L. Let $y \in F$. Since $y \leq x \vee y$, it follows that $x \vee y \in F$, that is, $y \in x^{-1} F$. Hence $F \subseteq x^{-1} F$, this completes the proof.

Proposition 3.4. Let $F$ and $G$ be filters of $L$. Then

(i) $x^{-1} F=L$ if and only if $x \in F$,

(ii) $x \leq y$ in $L \Rightarrow x^{-1} F \subseteq y^{-1} F$,

(iii) $F \subseteq G \Rightarrow x^{-1} F \subseteq x^{-1} G$,

(iv) $x^{-1}(F \cap G)=x^{-1} F \cap x^{-1} G$ and $x^{-1}(F \cup G)=x^{-1} F \cup x^{-1} G$,

(v) $(x \vee y)^{-1} F=x^{-1}\left(y^{-1} F\right)$,

(vi) $(x \wedge y)^{-1} F \subseteq x^{-1} F \cap y^{-1} F$,

for all $x, y \in L$.

Proof. (i) If $x \in F$, then $x \vee y \in F$ for all $y \in L$, that is, $y \in x^{-1} F$. Hence $x^{-1} F=L$. Conversely, assume that $x^{-1} F=L$. Then $x \vee y \in F$ for all $y \in L$, in particular $x=$ $x \vee x \in F$.

(ii) Assume that $x \leq y$ in $L$ and let $z \in x^{-1} F$. Then $x \vee z \in F$ and $x \vee z \leq y \vee z$. It follows that $y \vee z \in F$, that is, $z \in y^{-1} F$.

(iii)-(vi) Clear.

Definition 3.5 (see [2, Definition 4]). A proper filter $P$ of $L$ is said to be prime if for every $x, y \in L, x \vee y \in P$ implies $x \in P$ or $y \in P$.

Proposition 3.6. Let $P$ and $F$ be filters of $L$ such that $F \subseteq P$. If $P$ is prime, then $x^{-1} F \subseteq P$ for all $x \in L \backslash P$.

Proof. Let $z \in x^{-1} F$ for all $x \in L \backslash P$. Then $x \vee z \in F \subseteq P$. Since $P$ is prime, it follows that $z \in P$ because $x \notin P$. Hence $x^{-1} F \subseteq P$.

Proposition 3.7. If $P$ is a prime filter of $L$, then $L \backslash P$ is $\vee$-closed, that is, $x \vee y \in L \backslash P$ whenever $x \in L \backslash P$ and $y \in L \backslash P$.

Proof. The proof is straightforward.

The following theorem gives a characterization of prime filters.

THEOREM 3.8. A filter $P$ of $L$ is prime if and only if $x^{-1} P=P$ for all $x \in L \backslash P$. 
Proof. Suppose $P$ is a prime filter of $L$ and let $x \in L \backslash P$. The inclusion $P \subseteq x^{-1} P$ follows from Proposition 3.3. Let $y \in x^{-1} P$. Then $x \vee y \in P$ and so $y \in P$ because $P$ is prime and $x \notin P$. This proves that $x^{-1} P=P$. Conversely, assume that $x^{-1} P=P$ for all $x \in L \backslash P$. Let $y \vee z \in P$ and $z \notin P$. It follows from the hypothesis that $z^{-1} P=P$ so that $y \in z^{-1} P=P$. This shows that $P$ is prime.

Proposition 3.9. If $F$ is a filter of $L$, then $F=x^{-1} F \cap\langle F \cup\{x\}\rangle$ for all $x \in L \backslash F$.

Proof. Clearly, $F \subseteq x^{-1} F \cap\langle F \cup\{x\}\rangle$. Let $y \in x^{-1} F \cap\langle F \cup\{x\}\rangle$. Then $x \vee y \in F$ and $y \in\langle F \cup\{x\}\rangle$. It follows from Proposition 3.1 that there exists a nonnegative integer $n$ such that $n(x) y \in F$. Now

$$
n(x) y=x((n-1)(x) y)=(x \vee(n-1)(x) y)(n-1)(x) y .
$$

Since $y \leq(n-1)(x) y$, therefore $x \vee y \leq x \vee(n-1)(x) y$ and so $x \vee(n-1)(x) y \in F$. From $n(x) y=(x \vee(n-1)(x) y)(n-1)(x) y \in F$ it follows that $(n-1)(x) y \in F$. Continuing this process, we get $y \in F$ and, consequently, $x^{-1} F \cap\langle F \cup\{x\}\rangle \subseteq F$. This completes the proof.

Finally, we provide the prime filter theorem. This is a generalization of Liu and Xu's result [2, Theorem 4$]$ because every lattice ideal is necessarily $\vee$-closed.

THEOREM 3.10 (prime filter theorem). Let $F$ be a filter of $L$ and $S$ a $\vee$-closed subset of $L$ such that $F \cap S=\varnothing$. Then there exists a prime filter $P$ of $L$ such that $F \subseteq P$ and $P \cap S=\varnothing$.

Proof. The existence of a filter $P$ being the maximal element of the family of all filters that contain $F$ and have empty intersection with $S$ follows from an application of Zorn's lemma. We now prove that $P$ is prime. Suppose $P$ is not prime. By Theorem 3.8, there exists an element $x \in L \backslash P$ such that $x^{-1} P \neq P$. Now $P$ is properly contained in both $x^{-1} P$ and $\langle P \cup\{x\}\rangle$; therefore the maximality of $P$ implies that $x^{-1} P \cap S \neq \varnothing$ and $\langle P \cup\{x\}\rangle \cap S \neq \varnothing$. Let $y \in x^{-1} P \cap S$ and $z \in\langle P \cup\{x\}\rangle \cap S$. Then $y \in x^{-1} P$ and $z \in\langle P \cup\{x\}\rangle$ and hence $y \vee z \in x^{-1} P \cap\langle P \cup\{x\}\rangle=P$ by Proposition 3.9. Also $y \vee z \in S$ because $S$ is $\vee$-closed. Consequently, $y \vee z \in P \cap S$ and so $P \cap S \neq \varnothing$, a contradiction. This completes the proof.

\section{REFERENCES}

[1] Y. B. Jun, Implicative filters of lattice implication algebras, Bull. Korean Math. Soc. 34 (1997), no. 2, 193-198. MR 98g:03142. Zbl 876.03035.

[2] J. Liu and Y. Xu, On prime filters and decomposition theorem of lattice implication algebras, J. Fuzzy Math. 6 (1998), no. 4, 1001-1008. MR 2000b:03228. Zbl 920.03064.

[3] Y. Xu, Lattice implication algebras, J. Southwest Jiaotong Univ. (1993), no. 1, 20-27. Zbl 784.03035.

[4] Y. Xu and K. Y. Qin, On filters of lattice implication algebras, J. Fuzzy Math. 1 (1993), no. 2, 251-260. MR 94b:06016. Zbl 787.06009.

Young Bae Jun: Department of Mathematics Education, Gyeongsang National UniVERSITY, CHINJU 660-701, KOREA

E-mail address: ybjun@nongae.gsnu.ac.kr 


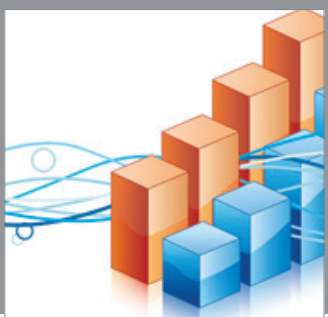

Advances in

Operations Research

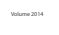

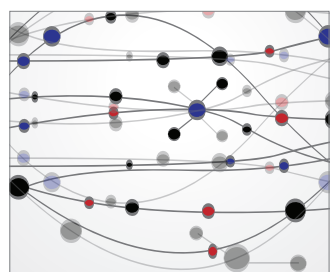

\section{The Scientific} World Journal
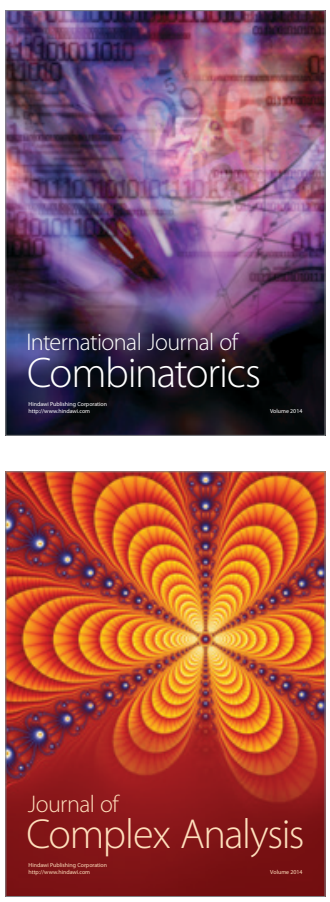

International Journal of

Mathematics and

Mathematical

Sciences
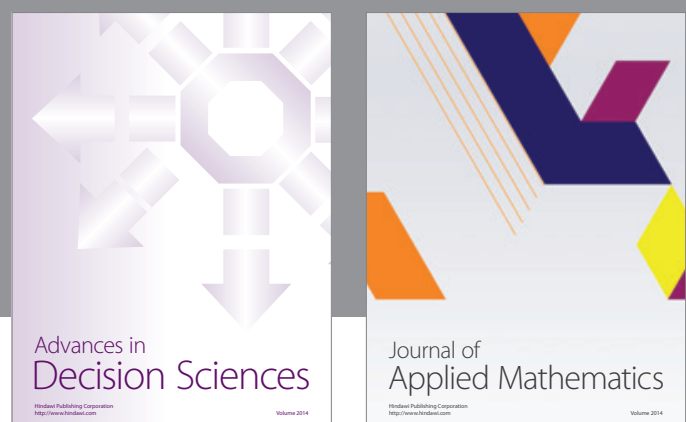

Journal of

Applied Mathematics
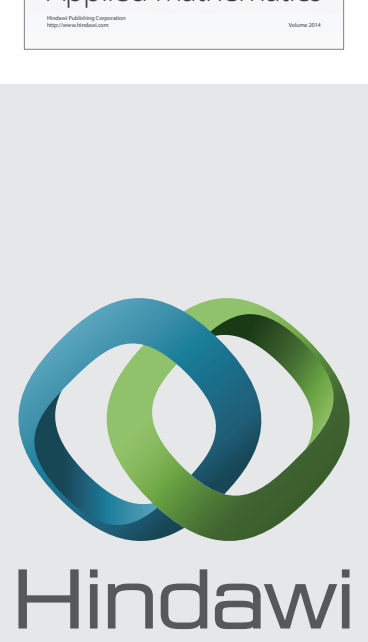

Submit your manuscripts at http://www.hindawi.com
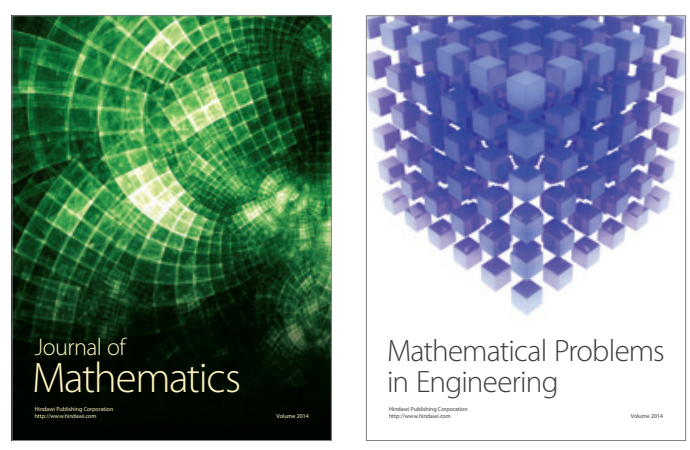

Mathematical Problems in Engineering
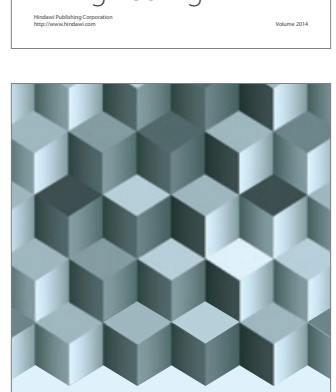

Journal of

Function Spaces
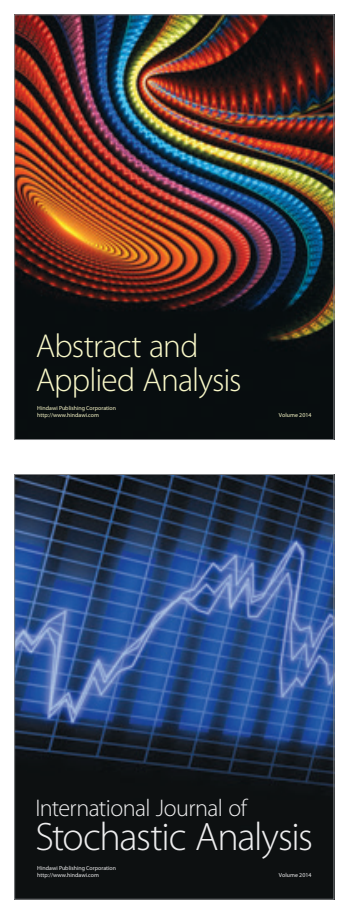

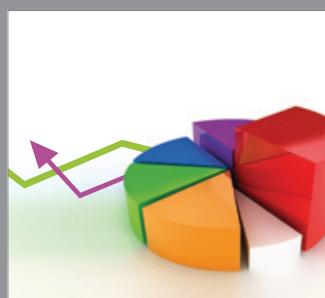

ournal of

Probability and Statistics

Promensencen
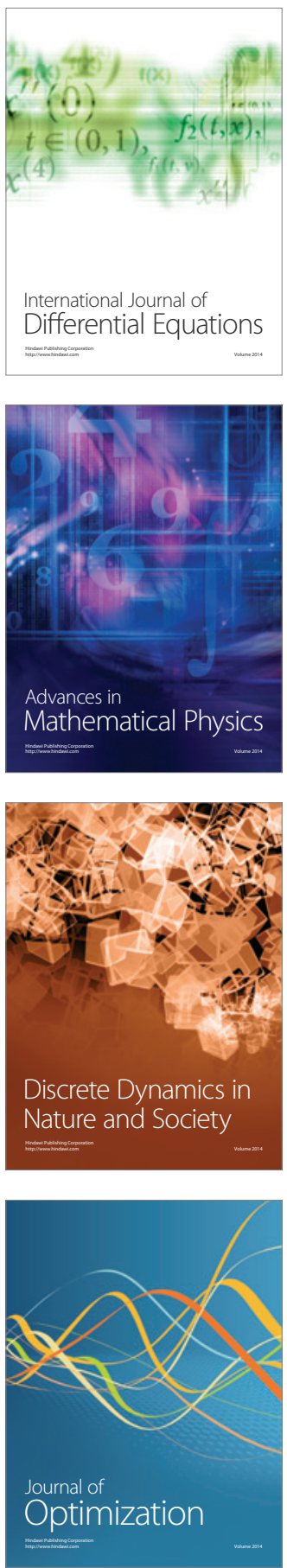